\title{
On the Need of Clinical and Pathological Investigation into the Nature and Causes of the Common Gynæcological Ailments.
}

\author{
By W. E. Fotrergili, M.A., B.Sc., M.D., Manchester.
}

TuE commonest gynæcological conditions are doubtless those which cause patients to complain of one or more symptoms such as menorrhagia, metrorrhagia, leucorrhøa, back-ache, bearing-down pain, pelvic discomfort, pain in the so-called ovarian regions, pains down the legs, reflex pain under the heart and the like. Examination in these conditions may reveal no physical signs, or may show that the uterus is merely soft and large, unduly firm, small and hard, or large and hard. The organ may be found in a position of retroversion, and, in addition, its axis may be bent backwards in retroflexion; or it may be anteverted and also anteflexed.

We have no accurate knowledge of the causation of these conditions. Indeed, the perusal of some recent work suggests that, in the pathology of minor gynæcological ailments, we have hardly advanced since the days of Plato, when the uterus was thought to wander about the body like a wild beast, which could sometimes be lured into the vagina; and when the "smoke test" was applied, not to drains, but vaginally, as an aid in the diagnosis of pregnancy. (Ballaniyne.)

Modern gynæcology is about one century old, and, during the last hundred years, the pathology of the commoner ailments may be said to have passed through two phases.

\section{The Meghanicat Phase.}

In making their observations, our ancestors relied mainly on vaginal examination, "bimanual" palpation being a device of comparatively recent date. The physical signs most easily recognized by a finger in the vagina were naturally variations in the position of the cervix and alterations in the shape of the uterus. If the fundus could be felt either in front of or behind the cervix, it was thought to be out of place; and if any curve, forward or backward, could be discovered, the orgen was regarded as misshapen. These most obvious physical signs were generally regarded as the actual lesions causing the symptoms complained of in each case, and thus the pathology of the commoner troubles of women entered its first or mechanical phase. The displacement theory has had a long and vigorous life, and is still living, though its field is

- Read at a meeting of the Pathological Society of Manchester on Feb. 12/h, 1908. 
now limited. For improved methods of examination quickly revealed the true nature of many grave conditions in which uterine displacements are observed. Thus it was realized that pelvic cellulitis may first push the uterus to one side and then pull it to the other; and that pelvic peritonitis may first push the organ forward and then pull it backward; while hæmatocele and hæmatoma were found to cause similar uterine movements.

The field of the displacement theory was thus considerably narrowed, and, later, doubt was thrown upon its validity as an explanation even of minor gynæcological troubles. Soon after the middle of last century Goupil* wrote as follows:-_." In later times the opinion was held that uterine deviations caused pain and grave functional disturbance; but Cruveilhier and Paul Dubois threw such doubt upon it, that even Valleix (1852), the last defender of the older views, says: 'some deviations of the uterus, both congenital and acquired, are unaccompanied by any pain or morbid symptom.' M. Depaul (1854) goes further, and in his report, declares that there are but a few rare cases in which the uterine deviation is of any consequence or requires direct treatment." These utterances, it will be noted, have quite a modern sound, though they were written fifty years ago.

Subsequently, it was gradually realized that moderate anteflexion is the typical shape, and that anteversion is the typical position of the uterus; but it was only about twenty years ago that Schultze and others succeeded in showing that practically all positions are normal in which the fundus lies in front of the pelvic axis and near the middle plane of the body.

Thus the sphere of utility of the displacement theory was still further reduced, and the waning mechanical phase in gynæcological pathology $\mathrm{dwindled}$ into comparative insignificance. Meanwhile the discovery of the part played by micro-organisms in the causation of disease was paving the way for the second phase, which waxed with the growth of the science of bacteriology, and which has largely held the field up to the present time to the almost total exclusion of what Goupil called " the older views."

\section{The Infectron Phase.}

Many gynæeological troubles of the kind we are considering have for long been known by names such as metritis, endometritis, endocervicitis, ovaritis and the like, and have long been described to the public as due to "inflammation." Soon after the entrance of the micro-organism on the pathological stage, it became clear that some conditions bearing these names are infective in origin. This discovery appears to have led many writers on gynæcology to forget

" "Clinical Memoirs on the Disenses of Women." Bernutz and Goupil. (New Sydenham Society, 1867, vol. ii, page 169.) 
their logic, and, arguing from the part to the whole, to conclude that all conditions with names ending in -itis must owe their origin to infection by one organism or another. Thus the pathology of the common diseases of women passed into its second or "infection" phase, and acquired thereby an air of fictitious and delusive simplicity. For the infective theory supplied a new and attractive explanation for almost every condition in the range of minor gynæcology. Nor did it only provide something to talk about; it also indicated a line of action. No Ionger was the pessary the only thing, for the whole range of antiseptic substances quickly became available for administration by the douche can and the plug. Thus patients passed from the frying-pan of displacement into the fire of infection; from the sway of the pessary into the swish of the disinfectant.

To those readers, and they will be many, who consider that this statement is too strong, the writer would recommend a perusal of recent works on gynæcology. Such a course of reading shows that many authors still consider that the majority of cases of so-called endometritis, metritis, ovaritis and the like, are of infective origin, and that they give practically no explanation of the nature of the remainder. Some authors advise that women should wear closed bifurcated garments with the object of preventing the entrance of germs into the vulva and vagina, so convinced are they that endometritis is caused by accidents of this kind. Others deprecate the use of the harmless necessary bath, especially near the end of pregnancy, lest water contaminated by the general surfase of the body should enter and infect the genital canal. This idea, by the way, is not new; for many years ago the belief was prevalent in bucolic circles that disaster was sure to occur to any female animal of the bovine race which was allowed to stand in water deep enough to reach the vulva.

We constantly see patients with menorrhagia, metrorrhagia, leucorrhoea, backache, "ovarian" pain and the like, and with alterations in the size and consistence of the uterus, whose condition is not to be explained by the infective any more than by the mechanical hypothesis. Some of these patients are young girls, whose symptoms date from puberty; others are young women, whose troubles date from marriage or from some period of general ill-health. In most of these there is nothing to suggest an infective origin for the pelvic condition. Many cases in which parturition or abortion has been the starting point are equally free from any indication of infection. The same may be said of most cases in which the symptoms appear near the change of life.

Not only do the conditions from which these patierts suffer receive names ending in -itis, but they are often regarded as the same in nature as lesions in which the symptoms date from an attack of gonorrhœe, a septic abortion or a puerperal infection. Our patho- 
logy, in fact, often falls below the level of that of the student of medicine who defined inflammation as anything beginning with pain, heat, redness and swelling, and ending in -itis. For it is often taken for granted that the conditions under discussion are inflammatory in nature and infective in origin, simply because their names end in -itis. It is not suggested that this view is taken by men who have had time to gain a knowledge of their profession by clinical experience; but it is maintained that many writers and teachers have neglected to instruct students about those conditions whose causation is not due to infection, while devoting ample attention to acute, sub-acute and chronic infective processes.

\section{Problems por Soldtion.}

The problems presented for solution by the non-infective minor ailments of women are by no means simple, and it may be well to indicate briefly their nature. First, it must be remembered that the uterus and its appendages have a life history which is much more complicated than that of other organs. Thus the anatomical and physiological changes which occur at puberty demand further investigation. The increase in muscular and vascular tissue relative to the uterine connective tissue doubtless begins some years before, and continues for some years after, the actual onset of menstruation. For during childhood the uterus contains about half as much muscle as it does connective tissue; during adolescence there is a relative increase of muscle until when womanhood is reached the proportions are reversed. This relative overgrowth of muscle is accompanied by an increase in vascular supply, both tissue changes being presided over by those intangible factors which determine the complex processes of development in living beings. That changes of this kind should go on regularly and without incoordination in the majority of human females, in spite of the artificial conditions of civilized life, is surely more wonderful than that in a few individuals the process should be upset. If, for instance, the growth of the uterine bloodvessels outpaces for a time the growth of the muscle, hyperæmia is the result. For the blood supply of the uterus is controlled and determined very largely by the action of its muscle fibres. This failure of the muscle to grow with the vessels is doubtless the true pathology of menorrhagia at and soon after puberty. Softening and enlargement of the organ follows if the muscle does not in a few months regain its ascendancy. If the condition persists long enough, fibrosis supervenes, as a result of prolonged venous congestion. The retrogressive tissue changes of the menopause also demand consideration. For some years before and after the actual cessation of menstruation there is a loss of muscle accompanied by obliterative changes in the blood-vessels and by relative increase of connective tissue. The changes which occur in the vascular walls render the 
blood supply less amenable to control by the surrounding muscle itself, a waning tissue, and the situation is often further complicated by the occurrence of vascular changes due to disease, local or general, in addition to the changes characteristic of and proper to the climacteric. Thus hæmorrhage near the menopause, with or without obvious changes in the consistence of the uterus, may ind icate either that the natural muscular atrophy is in advance of the natural arterial degeneration, or that pathological changes are present. Anomalies at the menopause are thus of interest alike to the clinician and to the pathologist.

The life history of the ovary must also be kept in mind, for deviations from its normal course must be recognized as factors in ætiology. Anomalies in the function of ovulation are the physical basis of many painful conditions which are generally called ovaritis or even peritonitis. It must, of course, not be forgotten that a genuine pelvic peritonitis, very mild in type, but capalle of producing extensive adhesions, is often set up by the free escape of blood from a hyperæmic ovary accompanying the rupiture of a Graafian follicle. Pronounced varicosity of the veins which supply the reproductive organs is by no means rare, and this lesion is doubtless the cause, in many cases, of pain which may be compared to that of varicocele in the male. Further, prolonged venous congestion probably can determine sclerosis of the ovary, which in turn causes pain during both regular and irregular periods of pelvic hyperæmia, and interferes with the ripening, rupture and healing of the Graafian follicles.

The changes which occur in connexion with pregnancy, parturition and involution, are even more striking than those which accompany the onset, the recurrence and the cessation of ovulation and menstruation. The enormous and rapid hypertrophy of pregnancy, the intense activity of labour and the rapid atrophy of the puerperium are processes whose mechanism may be thrown out of gear by very slight causes. The changes in any tissue involved may be either inadequate or excessive. Anomalies in the musculo-vascular relations frequently arise which are similar to, but more pronounced than those observed at puberty and the menopause, and which set up secondary changes not only in the reproductive organs, but in every pelvic organ and tissue. The results of such changes are far wider and more far-reaching than the usual paragraphs on "sub-involution" and "super-involution" would lead us to suppose.

We have thus noted several variations from the normal lifo history of the reproductive organs which are primary factors in much pelvic disease, and which demand extended investigation. There are other factors which often disturb the nervous control and the vascular supply of these organs, and originate both symptoms and morbid tissue changes. Thus in many cases the symptom; date not 
from puberty, parturition or the menopause, but from some period of general disease. A good example is afforded by anmmia, which, though often marked by relative amenorrhoea, is also frequently a cause of menorrhagia and metrorrhagia. The lesion which causes these symptoms is degeneration of the uterine muscle which, like degeneration of the cardiac muscle, occurs in many cases of anæemia. It is also recognized that all causes of backward pressure can aggravate, and at times can originate pelvic congestion sufficiently pronounced to produce symptoms and tissue changes which can be recognized by clinical methods.

In another group of cases the symptoms date from marriage, the commencement of sexual activity, apart from the occurrence of pregnancy, being enough in some predisposed persons to disturb the nervous control and the vascular supply of the uterine and ovarian tissue. In these cases it is doubtless seldom that actual physical changes are originated. More often, perhaps, pre-existent conditions are discovered soon after marriage, because the aggravation of symptoms due to married life first leads the patient to submit to examination.

Yet another problem is the real relationship between retroversion and uterine disease. The question may be restated as follows. The uterus is held in its typical position of anteversion by the lateral masses of blood-vessels, lymphatics, and perivascular connective tissue, which are attached to the sides of the cervix and lie below the bases of the broad ligaments and above the lateral vaginal fornices. These masses pass downwards and forwards from the sides of the pelvis practically in the line of the anterior branch of the internal iliac artery, and when normal they keep the cervix up and back in a manner which makes permanent retroversion impossible. If, however, the perivascular sheaths of the uterine vessels are relaxed and elongated, the cervix is abnormally free to move. The fundus may then tumble over into the pouch of Douglas, and is free to remain in a position of retroversion. In this position, the mass of tissue conveying the uterine blood supply is twisted through an angle of about $180^{\circ}$, and this twist is probably sufficient, in some persons, to check the venous return from the uterus without interfering with its arterial supply. While seldom able to produce, unaided, either symptoms or physical signs, the congestion thus produced by retroversion, appears able, when co-operating with other factors, to produce softening and enlargement of the uterus followed by retroflexion, by various changes in the cervix, and ultimately by fibrosis. So many authorities now doubt the truth of the above view that the question must still be regarded as open and calling for investigation; but this re-statement may perhaps prove to contain the remnant of solid truth which has survived the rest of the "displacement theory," and which still justifies the direct treatment of retroversion in certain cases. 